Washington University School of Medicine Digital Commons@Becker

Open Access Publications

2017

\title{
Quantitative FDG-PET/CT predicts local recurrence and survival for squamous cell carcinoma of the anus
}

\author{
Michael L. Cardenas \\ University of California, Davis \\ Christopher R. Spencer \\ Washington University School of Medicine in St. Louis \\ Stephanie Markovina \\ Washington University School of Medicine in St. Louis \\ Todd A. DeWees \\ Washington University School of Medicine in St. Louis \\ Thomas R. Mazur \\ Washington University School of Medicine in St. Louis
}

See next page for additional authors

Follow this and additional works at: https://digitalcommons.wustl.edu/open_access_pubs

Please let us know how this document benefits you.

\author{
Recommended Citation \\ Cardenas, Michael L.; Spencer, Christopher R.; Markovina, Stephanie; DeWees, Todd A.; Mazur, Thomas R.; \\ Weiner, Ashley A.; Parikh, Parag J.; and Olsen, Jeffrey R., "Quantitative FDG-PET/CT predicts local \\ recurrence and survival for squamous cell carcinoma of the anus." Advances in radiation oncology. 2, 3. \\ 281-287. (2017). \\ https://digitalcommons.wustl.edu/open_access_pubs/6169
}

This Open Access Publication is brought to you for free and open access by Digital Commons@Becker. It has been accepted for inclusion in Open Access Publications by an authorized administrator of Digital Commons@Becker. For more information, please contact vanam@wustl.edu. 
Authors

Michael L. Cardenas, Christopher R. Spencer, Stephanie Markovina, Todd A. DeWees, Thomas R. Mazur, Ashley A. Weiner, Parag J. Parikh, and Jeffrey R. Olsen 


\title{
Quantitative FDG-PET/CT predicts local recurrence and survival for squamous cell carcinoma of the anus
}

\author{
Michael L. Cardenas MD ${ }^{a}$, Christopher R. Spencer MD, MS ${ }^{b}$, \\ Stephanie Markovina MD, PhD ${ }^{\text {b }}$, Todd A. DeWees PhD ${ }^{\text {b }}$, \\ Thomas R. Mazur PhD ${ }^{\mathrm{b}}$, Ashley A. Weiner MD ${ }^{\mathrm{b}}$, Parag J. Parikh MD ${ }^{\mathrm{b}}$, \\ Jeffrey R. Olsen MD ${ }^{c, *}$
}

\author{
a Department of Radiation Oncology, University of California, Davis, California \\ ${ }^{\mathrm{b}}$ Department of Radiation Oncology, Washington University School of Medicine, St. Louis, Missouri \\ c Department of Radiation Oncology, University of Colorado School of Medicine, Denver, Colorado
}

Received 25 October 2016; received in revised form 10 March 2017; accepted 20 April 2017

\begin{abstract}
Purpose: ${ }^{18}$ F-fluorodeoxyglucose (FDG) positron emission tomography-(PET)/computed tomography (CT) imaging is used for staging and treatment planning of patients with anal cancer. Quantitative pre- and posttreatment metrics that are predictive of recurrence are unknown. We evaluated the association between pre- and posttreatment FDG-PET/CT parameters and outcomes for patients with squamous cell carcinoma of the anus (SCCA).

Methods and materials: The records of 110 patients treated between 2003 and 2013 with definitive radiation therapy for SCCA were reviewed under an institutional review board-approved protocol. The median radiation therapy dose was 50.4 Gy (range, 35-60 Gy). Concurrent chemotherapy was administered for 109 of 110 patients and generally consisted of 5-fluorouracil and mitomycin $\mathrm{C}(\mathrm{n}=94)$. All patients underwent pretreatment FDG-PET/CT and 101 of 110 underwent posttreatment FDG-PET/CT 3 months after completion of radiation therapy. The maximum standard uptake value $\left(\mathrm{SUV}_{\max }\right)$ was analyzed, in addition to multiple patient and treatment factors, by univariate and multivariate Cox regression for correlation with local recurrence (LR) and overall survival (OS).

Results: The median follow-up was 28.6 months. LR occurred in 1 of $15(6.7 \%), 5$ of $47(10.6 \%)$, and 6 of $48(12.5 \%)$ patients with stage I, II, and III disease, respectively. On univariate analysis, a significant association was observed between reduced LR and posttreatment $\mathrm{SUV}_{\max }<6.1(P=$ $.0095)$ and between increased OS and posttreatment $\mathrm{SUV}_{\max }<6.1(P=.0086)$. On multivariate analysis, a significant association was observed between reduced LR and posttreatment $\mathrm{SUV}_{\max }$ $<6.1(P=.0013)$ and the use of intensity modulated radiation therapy $(P<.001)$. A
\end{abstract}

\footnotetext{
Sources of support: There is no financial support to report.

Conflicts of interest: There are no conflicts of interest to report.

* Corresponding author. Department of Radiation Oncology, University of Colorado School of Medicine, 1665 Aurora Court, Room 1032, Aurora, CO 80045 .

E-mail address: jeffrey.r.olsen@ucdenver.edu (J.R. Olsen)
} 
significant multivariate association was observed between increased OS and posttreatment $\mathrm{SUV}_{\max }$ $<6.1(P=.0373)$ and the use of 5-fluorouracil/mitomycin $\mathrm{C}$ chemotherapy $(P=.001)$.

Conclusion: Posttreatment $\mathrm{SUV}_{\max }<6.1$ is associated with reduced LR and increased OS after chemoradiation therapy for SCCA independent of $\mathrm{T}$ and $\mathrm{N}$ stage on multivariate analysis. Greater follow-up is required to confirm this association with late patterns of failure.

(C) 2017 the Authors. Published by Elsevier Inc. on behalf of the American Society for Radiation Oncology. This is an open access article under the CC BY-NC-ND license (http:// creativecommons.org/licenses/by-nc-nd/4.0/).

\section{Introduction}

Anal cancer is a rare malignancy of the gastrointestinal tract with an annual incidence of 2.04 to 2.06 per 100,000 in the United States for men and women, respectively. Squamous cell carcinoma of the anus (SCCA) accounts for approximately $90 \%$ of primary anal malignancies. ${ }^{2}$ Known risk factors for SCCA are HIV and human papilloma virus infections, immunodeficiency, and smoking.

The combination of chemotherapy, consisting of 5-fluorouracil (5-FU) and mitomycin-C (MMC), and radiation has emerged as the standard of care for SCCA, with surgery reserved for salvage therapy. ${ }^{3-8}$ Radiation Therapy Oncology Group (RTOG) 0529 was a phase 2 trial that showed a significant reduction in grade $2+$ hematological and grade $3+$ gastrointestinal toxicities with the use of a dose-painted intensity modulated radiation therapy (DP-IMRT) regimen compared with the conventional regimen used in RTOG 9811. ${ }^{9}$ The reduced toxicity observed in RTOG 0529 has led to the adoption of IMRT as the standard of care. ${ }^{10}$

Previous studies have shown that $\mathrm{T}$ and $\mathrm{N}$ stages are prognostic factors that are correlated with overall and disease-free survival and that risk of nodal disease is directly associated with more advanced $\mathrm{T}$ stage. ${ }^{11}$ Furthermore, most treatment failures occur at the primary tumor site, followed by regional nodal failure. ${ }^{12,13}$

The role of ${ }^{18} \mathrm{~F}$-fluorodeoxyglucose (FDG)-positron emission tomography (PET)/computed tomography (CT) in the staging of SCCA has been evaluated by numerous studies. FDG-PET/CT has been shown to be a more sensitive imaging modality for detecting primary and nodal disease compared with CT. ${ }^{14,15}$ In one study, FDGPET/CT upstaged disease in $14 \%$ of cases and changed treatment planning in $17 \% .^{16} \mathrm{~A}$ previous report detailed the qualitative metabolic response on posttreatment FDGPET/CT and the association with cause-specific survival (CSS) and progression-free survival (PFS). ${ }^{17}$ The goals of this study were to evaluate the correlation of quantitative measures of FDG-PET/CT and IMRT treatment with local recurrence (LR) and overall survival (OS) and to compare recurrence outcomes both for quantitative measures and qualitative interpretation of the scans.

\section{Methods and materials}

\section{Patients}

In an institutional review board-approved study at Washington University in St. Louis, MO, the clinical records and PET/CT scans of patients who were treated with definitive radiation therapy for SCCA from 2003 to 2013 were retrospectively reviewed.

\section{Clinical staging}

Staging consisted of history and physical examination, digital rectal examination, anoscopy, and whole body FDG-PET/CT scans with a biopsy of the primary lesion and biopsy or fine-needle aspiration of the suspicious lymph nodes when clinically indicated.

\section{Treatment}

The method of radiation therapy delivery for SCCA transitioned from a 3-dimensional chemoradiotherapy (CRT) approach to IMRT during the course of the study period. Of the 110 patients in the study, 28 were treated conventionally and 82 with IMRT. Conventional treatment consisted of a 4-field box beam arrangement to the pelvis and photon and/or electron fields to the groin. To reduce the dose to the small bowel, femoral heads, bladder, iliac crest, large bowel, and external genitalia, IMRT plans were adopted. During the initial transition to IMRT, sequential plans were used. Generally, 30 Gy was delivered at 2 Gy per fraction for T1-2, T3, and T4 lesions. In the later years of the study, boost doses were incorporated into DP-IMRT fractions. With DP-IMRT, T2N0 disease was treated with 50.4 Gy and 42 Gy to the tumor and elective nodes, respectively, in 28 fractions. T3-4N0-3 disease was treated with 54 Gy to the tumor and 45, 50.4, and 54 Gy to the elective nodal, nodal disease $<3 \mathrm{~cm}$, and nodal disease $>3$ $\mathrm{cm}$, respectively, in 30 fractions.

A total of 94 patients received chemotherapy with 5-FU and MMC. This consisted of 2 cycles of 5-FU (1000 $\mathrm{mg} / \mathrm{m}^{2} / \mathrm{d}$ as a 96-hour infusion on days $1-5$ and days 
29-33 of DP-IMRT) and MMC (10 mg/m ${ }^{2}$ bolus on days 1 and 29). Eight patients received 5-FU only and 5 patients received 5-FU and cisplatin. One patient received 5-FU and octreotide and one received cisplatin and etoposide. Only one patient refused chemotherapy. A full list of patient characteristics is given in Table 1 .

\section{Follow-up}

Follow-up consisted of a physical and digital rectal examination and an anoscopy at 3 months initially after completion of treatment and then every 6 months for 5 years. Follow-up whole-body FDG-PET/CT was done at a median of 3.8 months after completion of treatment. Additional imaging was obtained annually or as clinically indicated. LR was defined as residual disease in the anal canal.

\section{${ }^{18} \mathrm{~F}$-fluorodeoxyglucose-positron emission tomography/computed tomography technique and image analysis}

FDG-PET/CT scans were performed on hybrid FDGPET/CT scanners (Biograph LSO 2, Biograph 40, Biograph Duo, Biograph mCT, Siemens Medical Solutions, Malvern, PA). CT scans were noncontrast images with $5-\mathrm{mm}$ slices taken from the skull base through the proximal thigh with $\mathrm{x}$-ray beam attenuation settings at 130 peak $\mathrm{kVp}$ and 110 effective mAs. PET images were taken at the same anatomical range and obtained 40 to 118 minutes after the administration of 11 to 20

Table 1 Patient characteristics

\begin{tabular}{ll}
\hline Characteristic & $\mathrm{n}(\%)$ \\
\hline Age & $17(15.4)$ \\
$\geq 65$ y & $93(84.6)$ \\
$\quad<65$ y & \\
Gender & $62(56.4)$ \\
$\quad$ Female & $48(43.6)$ \\
$\quad$ Male & \\
HIV Infection Status & $31(28.2)$ \\
$\quad+$ & $79(71.8)$ \\
$\quad-$ & \\
Tumor Stage & $64(58.2)$ \\
$1-2$ & $46(41.8)$ \\
3-4 & \\
Node Stage & $65(59.1)$ \\
0 & $45(40.9)$ \\
$1-3$ & \\
Chemotherapy & $94(85.5)$ \\
5-FU/MMC & $15(13.6)$ \\
Other & 1 \\
None & \\
\hline 5FU, 5-fluorouracil; MMC, mitomycin C.
\end{tabular}

$\mathrm{mCi}^{18} \mathrm{~F}(\mathrm{t} / 2=109.8$ minutes $)$ with imaging times of 2 to 4 minutes per bed position, depending on patient weight. Primary tumors were contoured and quantitative measures of the region of interest (ROI) were taken using MIMVista software (MIM Software Inc., Cleveland, $\mathrm{OH}$ ). The maximum standard uptake value $\left(\mathrm{SUV}_{\max }\right)$ of the ROI was used as the measure of avidity. Briefly, SUV is the ratio of the pixel intensity of the ROI divided by the hypothetical value of intensity where the radiotracer is distributed evenly throughout the body:

$\operatorname{SUV}(t)=\frac{\text { tissue activity }(t)}{\text { injected activity }(t) / \text { body mass }}$

$\Delta \mathrm{SUV}_{\max }$ was defined as the percentage change in $\mathrm{SUV}_{\max }$ from pretreatment to posttreatment FDG-PET/ CT. Radiology reports of the posttreatment FDG-PET/ CT scans were reviewed for qualitative data. On the basis of the reports, patients were classified as having complete response or incomplete response.

\section{Statistical analysis}

Survival was estimated using the Kaplan-Meier method. Univariate and stepwise multivariate Cox proportional hazard models were used to evaluate the association between variables of FDG-PET, radiation therapy modality, chemotherapy, $\mathrm{T}$ stage, $\mathrm{N}$ stage, and HIV with local recurrence-free survival (LRFS) and OS. Chemotherapy was categorized as "5-FU/MMC" and "Other" (including all other regimens). Cut-points were determined statistically for survival data using the outcomeoriented cut-point methodology defined by Contal and O'Quigley. ${ }^{18}$

Qualitative and quantitative measures were compared with the $\chi^{2}$ and Fisher's exact tests. The strength of association was quantified with Cohen's kappa coefficient. $P<.05$ was considered statistically significant. All tests were 2-sided. Statistical analyses were performed with StatView and SAS (Versions 5.0.1 and 9.2, respectively; SAS Institute Inc., Cary, NC).

\section{Results}

\section{Patient factors}

The records of 110 patients treated between 2003 and 2013 with definitive radiation therapy were reviewed. The average age at diagnosis was 54.5 years. The number of $\mathrm{HIV}+$ patients was $31(28.2 \%)$, of whom 25 were managed with antiretroviral therapy. CD4+ T cell counts were available at the time of treatment for 21 of these patients (range, 8-1031). A total of 109 patients received chemotherapy. A full list of patient characteristics is shown in Table 1. 


\section{Clinical outcomes}

The median follow-up was 28.6 months (range, 3.6-94.1 months). There were 12 LRs and 24 deaths among the 110 patients in the study. One of 15 patients with stage I disease (6.7\%), 5 of 47 patients with stage II disease (10.6\%), and 6 of 48 patients with stage III disease $(12.5 \%)$ had LR. Patients with and without LR received median radiation therapy doses of $50 \mathrm{~Gy}$ and $50.4 \mathrm{~Gy}$, respectively.

\section{${ }^{18} \mathrm{~F}$-fluorodeoxyglucose-positron emission tomography/computed tomography characteristics}

During pretreatment FDG-PET/CT, the mean $\mathrm{SUV}_{\max }$ was 11.73. Among patients with and without LR, the mean pretreatment $\mathrm{SUV}_{\max }$ was 9.97 and 11.95, respectively. The median time to posttreatment FDGPET/CT was 3.8 months. Mean posttreatment $\mathrm{SUV}_{\max }$ was 4.73 , and mean posttreatment $\mathrm{SUV}_{\max }$ among patients with and without LR was 5.35 and 4.66, respectively.

An $\mathrm{SUV}_{\max }$ of 6.1 was identified as the appropriate cut-point for survival analysis. Patients at or below this cut-point on the basis of posttreatment imaging were classified as $\mathrm{SUV}_{\max }$ low and those above were classified as $\mathrm{SUV}_{\max }$ high. Similarly, a $\Delta \mathrm{SUV}_{\max }$ of $-62.3 \%$ was identified as the appropriate cut-point for survival analysis.

During posttreatment FDG-PET/CT, 40 of 101 patients were reported as having a complete response qualitatively on the basis of a radiology review. All others were classified as having incomplete response. Twenty-two of twenty-six $\mathrm{SUV}_{\max }$ high patients were classified as having an incomplete response and 39 of $75 \mathrm{SUV}_{\max }$ low patients were classified as having a complete response.

\section{Radiation therapy technique}

The radiation therapy technique was 3-dimensional CRT for 28 patients and IMRT for 82 patients. The median follow-up for the 3-dimensional CRT group was 36 months versus 24 months for the IMRT group. The 3-dimensional CRT group had 8 LRs with a median time to recurrence of 12 months versus 4 LRs in the IMRT group, with a median time to recurrence of 20 months. Two-year LRFS was $72 \%$ for the 3-dimensional CRT and 95\% for the IMRT group $(P<.01)$. Recurrence-free survival was $86 \%$ for the IMRT group and $72 \%$ for the 3 -dimensional CRT group at 2 years $(P=.09)$. OS was $88 \%$ for both groups at 2 years $(P=.4)$. Within the IMRT group, there were 2 LRs each in the simultaneous integrated boost and the sequential boost groups.

\section{Univariate analysis}

On univariate analysis of FDG-PET/CT factors, a significant association was observed between improved LRFS and pretreatment $\mathrm{SUV}_{\max }>7.1$ (hazard ratio [HR] $=0.2788, P=.0296)$ and between improved LRFS and posttreatment $\mathrm{SUV}_{\max }<6.1(\mathrm{HR}=0.2276, P=.0172)$. A significant univariate association was also observed between increased OS and posttreatment $\mathrm{SUV}_{\text {max }}<6.1$ $(\mathrm{HR}=0.3050, P=.0127)$. Kaplan-Meier curves for posttreatment $\mathrm{SUV}_{\max }$ groups for LRFS and OS are shown in Figures $1 \mathrm{~A}$ and $\mathrm{B}$.

A $\Delta \mathrm{SUV}_{\max }$ of greater magnitude than $-62.3 \%$ was associated with improved OS (HR $=0.2283, P=.0080)$ but was not a significant predictor of LRFS on univariate analysis.

On univariate analysis of clinical factors, no correlation was found between HIV status and LR risk. The correlation between T/N stage and LR risk approached but did not reach a significance in this data set.

On univariate analysis of treatment factors, a significant association was observed between improved LRFS and the use of 5-FU/MMC chemotherapy (HR $=0.2079$, $P=.0114)$. This chemotherapy regimen was also significantly associated with increased OS on univariate analysis $(\mathrm{HR}=0.0811, P<.0001)$. Additionally, there was a significant association between improved LRFS and the use of IMRT compared with conventional radiation therapy (HR $=0.1839, P=.0058)$.

\section{Multivariate analysis}

On multivariate analysis, a significant association was observed between increased LRFS and posttreatment $\mathrm{SUV}_{\max }<6.1(\mathrm{HR}=0.1658, P=.0013)$ and the use of IMRT $(\mathrm{HR}=0.0930, P=.0002)$. The variables of 5 FU/MMC chemotherapy and pretreatment $\mathrm{SUV}_{\max }>7.1$ were not significant for LR on multivariate analysis.

A significant multivariate association was observed between increased $\mathrm{OS}$ and posttreatment $\mathrm{SUV}_{\max }<6.1$ $(\mathrm{HR}=0.3551, P=.0373)$. Similarly, a $\Delta \mathrm{SUV}_{\max }$ of greater magnitude than $-62.3 \%$ was associated with increased OS (HR $=0.3000, P=.0298) .5$-FU/MMC chemotherapy was strongly associated with increased OS $(\mathrm{HR}=0.1060, P<.0001)$.

\section{Categorical analysis}

On $\chi^{2}$ analysis and Fisher's exact test, there was a significant association between the qualitative and quantitative analyses of the FDG-PET/CT scan $(P=.0034$ and $P=.0047$, respectively) with a kappa value of 0.2265 (95\% confidence interval, 0.09-0.75), which indicates a fair association. ${ }^{19}$ The qualitative assessment did not reach significance in predicting LR. 
A Post-treatment PET/CT

Local Recurrence Free Survival

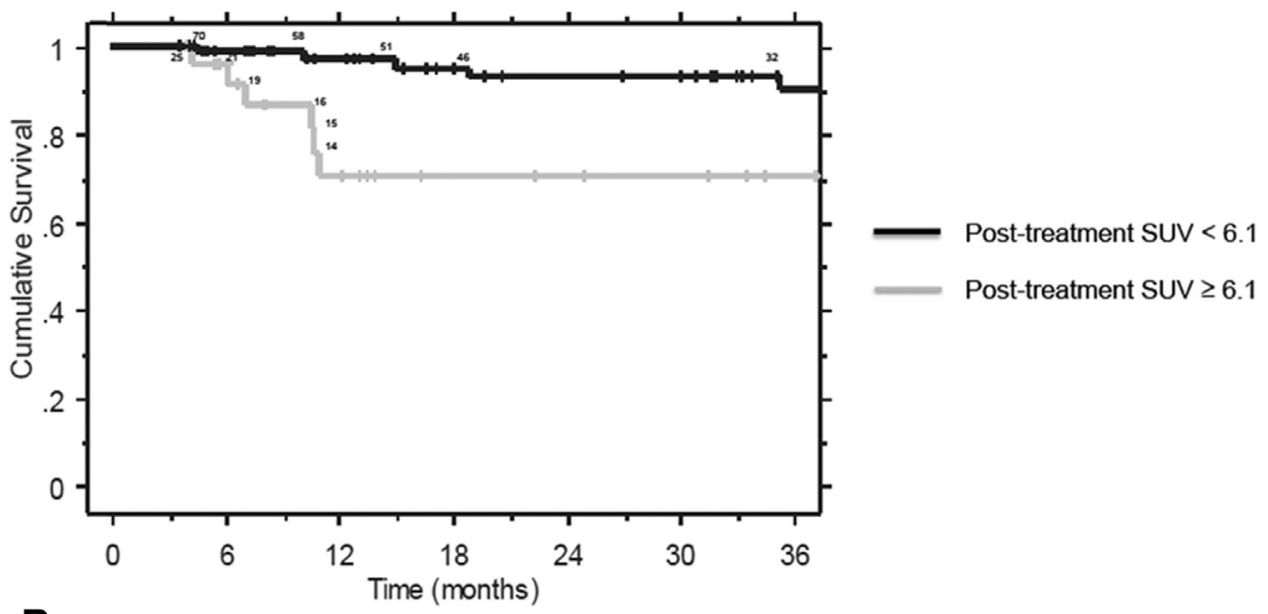

B

\section{Post-treatment PET/CT Overall Survival}

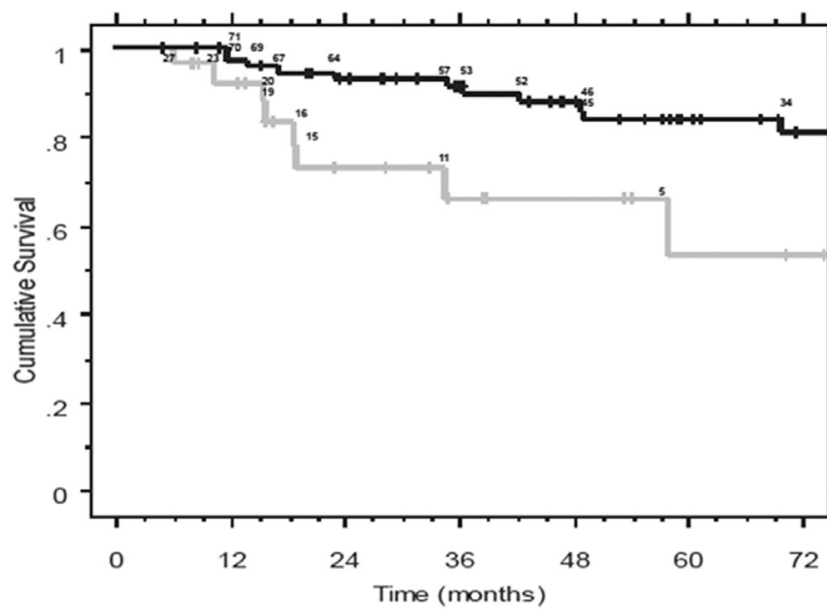

Figure 1 (A) Kaplan Meier survival analysis of Post-treatment SUVmax groups and Local Recurrence Free Survival. The number of patients at risk at the time of each local recurrence event is shown above the respective curves. (B) Kaplan Meier survival analysis of Post-treatment SUVmax groups and Overall Survival. The number of patients at risk at the time of each mortality event is shown above the respective curves.

\section{Discussion}

Most studies on the role of FDG-PET/CT in SCCA have focused on the diagnostic and treatment planning significance of this imaging modality. There is only limited literature on the independent and quantitative prognostic significance of FDG-PET/CT in SCCA. A previous study of FDG-PET/CT in anal cancer correlated metabolic response with patient outcomes. In the current study, 53 patients with SCCA received chemoradiation and had a staging FDG.

PET/CT and follow-up FDG-PET/CT occurred at a mean of 2.1 months after completion of therapy. At follow-up, patients were stratified as either having a complete metabolic response $\left(\mathrm{SUV}_{\max }\right.$ in the ROI no greater than blood pool SUV) or a partial response
$\left(\mathrm{SUV}_{\max }\right.$ in the ROI greater than blood pool SUV) as judged by the radiologist. The endpoints of the study were 2-year CSS and 2-year PFS. Patients with a complete metabolic response had a 2-year CSS and a 2-year PFS of $94 \%$ and $95 \%$, respectively. Patients with a partial metabolic response had 2-year CSS and 2-year PFS of $39 \%$ and $22 \%$, respectively. ${ }^{17}$ To the authors' knowledge, our study is the first to correlate the quantitative values of $\mathrm{SUV}_{\max }$ on pretreatment and posttreatment FDG-PET/CT with LRFS.

Our results suggest that in SCCA, a higher posttreatment $\mathrm{SUV}_{\max }$ is predictive of worse clinical outcomes. This finding is consistent with a previous qualitative study that evaluated tumors as complete or partial responders. We investigated the level of covariance between the qualitative reports on the basis of initial radiology reports 
and the quantitative data in this study. As expected, there is a significant correlation; however, the strength of this association was not robust. The majority of nonresponders on the basis of quantitative analysis (high posttreatment $\mathrm{SUV}_{\max }$ ) were also categorized as incomplete responders qualitatively. However, the responders on the basis of quantitative analysis (low posttreatment $\mathrm{SUV}_{\max }$ ) were inconsistently classified as such qualitatively. Overall, two-thirds of posttreatment FDG-PET/CT scans were read as incomplete response. This fact diluted the data set such that qualitative assessment failed to reach significance in predicting LR. In this context, a quantitative threshold for posttreatment tumor $\mathrm{SUV}_{\max }$ may be useful and potentially more specific for predicting LR.

The observation that higher $\mathrm{SUV}_{\max }$ during pretreatment FDG-PET/CT was associated with better clinical outcomes was unexpected and unusual. However, this finding was not significant on multivariate analysis and was likely due to a skewed number of patients in this group who did not receive 5-FU/MMC chemotherapy.

There are a number of potential weaknesses in this study. There is limited follow-up of patients at the later time points. It is possible that some of these patients had LR after the conclusion of the study period. This may partially explain the improvement observed in LRFS with the use of IMRT. Additionally, through the course of the study multiple PET/CT scanners were used, and it is possible that different scanners had variable quantifications of FDG uptake.

FDG uptake has been shown to be predictive of outcomes after treatment in a number of solid tumors and lymphoma. Most reports show that greater PET avidity is predictive of worse outcomes. Sakaki et al demonstrated that $\mathrm{SUV}_{\max }>5.0$ was associated with worse OS and disease-free survival in non-small cell lung cancer stages I-IIIb. ${ }^{20}$ Pan et al conducted a systematic review of the literature with regard to the prognostic significance of SUV in esophageal cancer. This group also found that a high pretreatment SUV was predictive of worse OS and disease-free survival. ${ }^{21}$

In lymphoma, PET avidity is positively correlated with serum lactate dehydrogenase, which is a prognostic marker, and more aggressive lymphomas typically have high SUV values compared with indolent lymphomas. FDG-PET/CT guide the management of lymphoma by enabling assessment of response to treatment in the posttreatment scenario and the identification of refractory disease during treatment. ${ }^{22}$

In work presented at the 2014 Annual Meeting of the American Society for Radiation Oncology, Mai et al reported a retrospective study of the influence of human papilloma virus status and $\mathrm{p} 16$ expression on outcomes in patients with anal cancer who were treated with chemoradiation therapy. ${ }^{23}$ Although this was not available for the patients in our study, future studies can evaluate the relationship between human papilloma virus infection status and PET avidity in both pre- and posttreatment scenarios.

\section{Conclusion}

Posttreatment $\mathrm{SUV}_{\max }<6.1$ is associated with increased LRFS and increased OS after chemoradiation therapy for SCCA independent of $\mathrm{T}$ and $\mathrm{N}$ stage on multivariate analysis. Our data suggest that the quantitative metric of $S_{U V}$ max may be more specific for LR than a qualitative assessment of FDG-PET/CT. Greater followup is required to confirm the association with late patterns of failure.

\section{References}

1. Johnson G, Madeleine MM, Newcomer LM, Schwartz SM, Daling JR. Anal cancer incidence and survival: The surveillance, epidemiology, and end results experience, 1973-2000. Cancer. 2004;101:281-288.

2. Fenger C, Frisch M, Marti MC, et al. Tumours of the anal canal. In: Hamilton SR, Aoltonene LA, eds. Pathology and Genetics of Tumours of the Digestive System. Lyon: IARC Press; 2000:145-155.

3. UKCCCR Anal Canal Cancer Trial Working Party. Epidermoid anal cancer: Results from the UKCCCR randomized trial of radiotherapy alone versus radiotherapy, 5-fluorouracil and mitomycin C. Lancet. 1996;348:1049-1054.

4. Bartelink H, Roelofsen F, Eschwege F, et al. Concomitant radiotherapy and chemotherapy is superior to radiotherapy alone in the treatment of locally advanced anal cancer: Results of a phase III randomized trial of the European Organization for Research and Treatment of Cancer Radiotherapy and Gastrointestinal Cooperative Groups. J Clin Oncol. 1997;15:2040-2049.

5. Flam M, John M, Pajak TF, et al. The role of mitomycin $\mathrm{C}$ in combination with 5-fluorouracil and radiotherapy, and of salvage chemoradiation in the definitive nonsurgical treatment of epidermoid carcinoma of the anal canal: Results of a phase III randomized Intergroup study. J Clin Oncol. 1996;14:2527-2539.

6. Ajani JA, Winter KA, Gunderson LL, et al. Fluorouracil, mitomycin, and radiotherapy vs fluorouracil, cisplatin and radiotherapy for carcinoma of the anal canal: A randomized controlled trial JAMA. 2008;299:1914-1921.

7. Gunderson LL, Winter KA, Ajani JA, et al. Long-term update of US GI Intergroup RTOG 98-11 phase III trial for anal carcinoma: Disease-free and overall survival with $\mathrm{RT}+5$-FU-mitomycin versus RT+5-FU-cisplatin. J Clin Oncol. 2011;29:4005.

8. James R, Wan S, Sebag-Montefiore D, et al. A randomized trial of chemoradiation using mitomycin or cisplatin, with or without maintenance cisplatin/5-FU in squamous cell carcinoma of the anus (ACT II). J Clin Oncol. 2009;27:LBA4009.

9. Kachnic LA, Winter K, Myerson RJ, et al. RTOG 0529: A phase 2 evaluation of dose-painted intensity modulated radiation therapy in combination with 5-fluorouracil and mitomycin-C for the reduction of acute morbidity in carcinoma of the anal canal. Int J Radiat Oncol Biol Phys. 2013;86:27-33.

10. National Comprehensive Cancer Network. Anal carcinoma. Version 1. 2016. Available at: http://www.ncen.org/professionals/physician gls/pdf/anal.pdf. Accessed December 21, 2015.

11. Myerson RJ, Outlaw ED, Chang A, et al. Radiotherapy for epidermoid carcinoma of the anus: thirty years' experience. Int $J$ Radiat Oncol Biol Phys. 2009;75:428-435. 
12. Das P, Bhatia S, Eng C, et al. Predictions and patterns of recurrence after definitive chemoradiation for anal cancer. Int J Radiat Oncol Biol Phys. 2007;68:794-800.

13. Wright JL, Patil SM, Temple LK, et al. Squamous cell carcinoma of the anal canal: Patterns and predictors of failure and implications for intensity-modulated radiation treatment and planning. Int J Radiat Oncol Biol Phys. 2010;78:1064-1072.

14. Mistrangelo M, Pelosi E, Bello M, et al. Role of positron emission tomography-computed tomography in the management of anal cancer. Int J Radiat Oncol Biol Phys. 2012;84:66-72.

15. Cotter SE, Grigsby PW, Siegel BA, et al. FDG-FDG PET/CT in the evaluation of anal carcinoma. Int J Radiat Oncol Biol Phys. 2006; 65:720-725.

16. Sveistrup J, Loft A, Berthelsen AK, Henriksen BM, Nielsen MB, Engelholm SA. Positron emission tomography/computed tomography in the staging and treatment of anal cancer. Int J Radiat Oncol Biol Phys. 2012;83:134-141.

17. Schwartz JK, Siegel BA, Dehdashti F, Myerson RJ, Fleshman JW, Grigsby PW. Tumor response and survival predicted by post-therapy FDG-FDG - PET/CT in anal cancer. Int J Radiat Oncol Biol Phys. 2008;71:180-186.

18. Contal C, O'Quigley J. An application of changepoint methods in studying the effect of age on survival in breast cancer. Comput Stat Data Analysis. 1999;20:253-270.

19. Fleiss JL. Statistical Methods for Rates and Proportions. 2nd ed. New York, NY: Jon Wiley; 1981.

20. Sasaki R, Komaki R, Macapiniac H, et al. [18-F] fluorodeoxyglucose uptake by positron emission tomography predicts outcome of non-small cell lung cancer. J Clin Oncol. 2005;23:1136-1143.

21. Pan L, Gu P, Huang G, Xue H, Wu S. Prognostic significance of SUV on FDG - PET/CT in patients with esophageal cancer: A systematic review and meta-analysis. Eur J Gastroenterol Hepatol. 2009;21:1008-1015.

22. Cronin CG, Swords R, Troung MT, et al. Clinical utility of PET/CT in lymphoma. Am J Roentgenol. 2010;194:W91-W103.

23. Mai SK, Reuschenbach M, Ottstadt M, et al. The influence of HPVstatus on outcome of anal cancer treated with radio-chemotherapy. Int J Radiat Oncol Biol Phys. 2014;90:S31-S32. 